\title{
Recorrido histórico de la alfabetización en Venezuela desde el desaprendizaje
}

\section{Historical journey of literacy in Venezuela from the un-learning}

\begin{abstract}
Joamsner Magdalena Arévalo es Profesora Agregada de la Universidad Nacional Experimental Francisco de Miranda (Venezuela) (Smartkelly1912@gmail.com) (iD (https://orcid.org/0000-000 1-7617-7265)
\end{abstract}

Recibido: 2018-04-07 / Revisado: 2019-05-13 / Aceptado: 2019-05-17 / Publicado: 2019-07-01

\section{Resumen}

Actualmente los ciudadanos están expuestos a una gran cantidad de información presentada a través de diversos medios, lo que exige destrezas que no solo se limiten a la reproducción y decodificación sino que los mismos deben ser capaces de interpretar y expresarse a través de distintos medios y en más de un idioma (Crystal, 20l I). En este sentido los conceptos relacionados con las prácticas alfabetizadoras alrededor del mundo se han ido transformando hacia un enfoque más humanista en el que la producción y comprensión del discurso escrito se aprecie como una herramienta de crecimiento personal incluyendo el uso de las Tecnologías de la Información Comunicación (TIC). Con esto en mente se expone un recorrido histórico del concepto de alfabetización en Venezuela iniciando con los programas de alfabetización fuera del contexto escolar con el fin de dar inicio a un estudio futuro más profundo que, enmarcado en los postulados del racionalismo crítico y el desaprendizaje (Andrade, 2005; Popper, 2008), lleve a reconstruir el concepto y reoriente las prácticas escolares de lectura y escritura. Este estudio se basó en una revisión documental de las fuentes disponibles en medios oficiales en relación con la enseñanza de la lectura y la escritura en Venezuela aportando como conclusión principal que no solo no hay un consenso sobre la visión y metodología para la alfabetización sino que el mencionado replanteamiento se hace necesario.

Descriptores: Alfabetización, recorrido, prácticas, concepto, racionalismo, desaprendizaje.

\begin{abstract}
Currently citizens are exposed to a large amount of information presented through various means. This torrent of information requires skills that are not only limited to the reproduction and decoding of signs and symbols but, they must be able to interpret and express themselves through different means and in more than one language. (Crystal, 20II) In this sense, the concepts related to literacy practices around the world have been transformed towards a more humanistic approach in which the production and understanding of written discourse is appreciated as a tool for personal growth, including in this the use of Information and Communication Technology (ICT). With this in mind, a historical journey of the concept of literacy in Venezuela is exposed in order to start a deeper study in the future,
\end{abstract}

Forma sugerida de citar: Arévalo, J. (2019). Recorrido histórico de la alfabetización en Venezuela desde el desaprendizaje. Alteridad, 14(2), 256-266. https://doi.org/10.17163/alt.v14n2.2019.09 
that reconstructs the concept of literacy and reorients the school practices of reading and writing in the country, through the frame of critical rationalism and unlearning (Andrade, 2005 y Popper, 2008). This study was based on a documentary review of the sources available in official media regarding the teaching of reading and

\section{Introducción}

La alfabetización es básicamente el acto de enseñar el manejo productivo de la lengua escrita. A través de esta escueta definición se puede apreciar lo que usualmente se practica en las aulas de clase durante el proceso de enseñanza y aprendizaje de la escritura, sin embargo es justo admitir que para que un individuo pueda llegar a comunicarse competentemente a través de la escritura primero debe poder entender que escribir y leer son formas de hacer cosas que están conectadas con su cotidianidad y no actividades que solo se hacen por requerimiento laboral o académico. Ser escritor o lector no se trata de ser autor de libros, se trata de ser una persona que se inserta en su comunidad por medio de la expresión escrita de sus ideas.

Para llegar a comprender esto, las prácticas actuales de alfabetización deberían pasar por un proceso de desaprendizaje y contrastación crítica (Andrade, 2005; Popper, 2008) que al reorientar el concepto de alfabetización promueva también la transformación del proceso de enseñanzaaprendizaje de la lectura y la escritura en los contextos escolares. Ya diversos autores han dado cuenta de las enormes deficiencias que reportan los egresados de educación primaria e incluso adultos que han participado en los distintos programas de alfabetización llevados a cabo por parte del gobierno venezolano en diferentes momentos de la historia, por lo que se expone a continuación una exploración inicial de los conceptos esenciales de alfabetización que se han contemplado en Venezuela desde las fuentes gubernamentales oficiales a través de los años, esto con el fin de dar un primer paso en la conducción de una investigación más profunda que writing in Venezuela, providing as a main conclusion that not only is there no consensus on the vision and methodology for literacy, but also the mentioned rethinking becomes necessary.

Keywords: Literacy, journey, practices, concept, rationalism, un-learning.

indague sobre las prácticas sociales de alfabetización en Venezuela.

\section{Alfabetizar. La reflexión como primer paso}

En el transcurso de su vida, el hombre adquiere, aprende, perfecciona y desecha infinidad de comportamientos, algunos heredados de su entorno social, tales como el habla y sus modismos culturales, otros aprendidos conscientemente en contextos educativos entre ellos la legua escrita. Esto lo lleva a una transformación de sí mismo que en muchos casos no se detiene hasta el final de su existencia.

En este orden de ideas, como seres sociales, los individuos no dejan de expresarse, y es la comunicación verbal, específicamente la escrita, el signo inequívoco de la condición humana. Así pues, entendemos por comunicación el acto de poner en sonidos, signos o símbolos ideas que van orientadas a un destinatario específico adaptadas a un contexto particular. Estos sonidos, signos o símbolos articulados configuran el lenguaje, el cual es un fenómeno dinámico revestido de creatividad, productividad y complejidad. A esto se pueden añadir las palabras de Ferreiro y Teberosky (1991) quienes afirman que son las reglas y parámetros de articulación los que definen un lenguaje, pues las palabras por sí solas no representan nada hasta que no son combinadas para formar oraciones que construyan un mensaje inteligible. A esta idea se suma Franco (2000) al expresar que "el lenguaje no tiene sentido si no es en la comunicación interhumana" (p. 83) y es sin duda la lengua escrita la expresión máxima del lenguaje articulado y regulado en el hombre. 
Ya diversos autores han enumerado las diferencias entre la lengua oral y la lengua escrita, sin embargo es oportuno citar a Harmer (2000) quien indica que, a diferencia de la lengua oral, la escrita debe ser aprendida; es decir requiere un proceso de instrucción formal que suele comenzar en la niñez, posterior a la adquisición del lenguaje oral. A este proceso se le denomina alfabetización y puede ser definido como el acto de aprendizaje, creación e interpretación del lenguaje escrito con el propósito de comunicarse.

En efecto, si se sostiene que el habla y la audición se desarrollan partiendo de la necesidad de expresarse y ser entendido, tiene mucha más relevancia esta necesidad cuando se refiere a la escritura, y es que es innegable la intención social de la lengua escrita, tomando las palabras de Cassany (1999) escribir o leer es una forma de usar el lenguaje para realizar acciones y conseguir objetivos. Leemos y escribimos con el objetivo de persuadir, argumentar, solicitar, hacer catarsis, crear arte, regular los comportamientos, entre otras cosas. El lenguaje es el medio y también el fin del desarrollo del pensamiento humano.

Entonces, aprender implica un cambio de conducta, así como la creación y desarrollo de nuevas estructuras cognitivas en el ser humano, y todo este proceso es exteriorizado y expresado ante el mundo a través del lenguaje. Igualmente, este motiva al individuo a cambiar su realidad, a experimentar nuevas formas de vivirla a través del lenguaje. Así pues, cualquier proceso de aprendizaje en el ser humano va estrechamente ligado con el desarrollo del lenguaje. Es de hecho el lenguaje el instrumento de aprendizaje, y en particular las palabras, son entonces, en ese afán del hombre por nombrar todo aquello que compone su ambiente, la forma que adquiere el pensamiento cuando se va transformando durante todo este proceso.

Se puede decir, para reafirmar esta idea, que el conocimiento es el producto del aprendizaje y que el lenguaje lo configura. Se llega así al punto en que, dada la naturaleza social del hombre, este busca transmitir a sus semejantes dicho conocimiento y en el proceso ampliarlo y perfeccionarlo. Es aquí donde la escritura se convierte en la modalidad de transmisión por excelencia, dada su naturaleza perdurable en el tiempo aún en momentos de preponderancia de medios audiovisuales y realidad virtual (Brown, 2007).

De hecho, tal como lo expone Cassany (2003) la escritura es y sigue siendo "el código más usado para interactuar en las comunidades virtuales". El hecho de que cada vez menos personas usen papel y lápiz para expresar sus ideas no le ha quitado vigencia a la escritura, por el contrario, las redes sociales, los blogs, y otras formas de comunicación vía internet han reforzado la importancia de la comunicación escrita, hasta el punto de que las personas llegan a crearse identidades que solo existen en palabras e imágenes pero que influencian a comunidades de personas hasta tal punto que trascienden a la realidad más allá de lo virtual.

Continuando con Cassany (2003), la comunicación mediatizada por ordenador (CMO) ha promovido el acercamiento a la información desde el punto de vista de la competencia multimodal, en la cual el individuo representa y aborda los mensajes desde una variedad de sistemas: habla, escritura, imagen, audio y cualquier combinación que sea permitida dentro del campo virtual, de modo que lo que comenzó como un documento escrito se convierte en una comunidad de expresión. De allí que si hubiese que reconcebir, la noción tradicional de escritura también corresponderá hacerlo con la de alfabetización. Ya no se puede limitar la alfabetización al manejo de los signos y símbolos mencionados al inicio, así como a las convenciones que regulan el lenguaje escrito; delimitándolo al uso del lápiz y papel, sino que se debe abarcar el uso y manejo de los medios audiovisuales para crear, representar, compartir, comprender e interpretar información en diversos medios de interacción humana y con distintos propósitos.

Con respecto a esto, se puede traer a colación lo señalado por la UNESCO (citado en 
Ortiz, 2007), cuando se refiere a la alfabetización como: la "habilidad para identificar, entender, interpretar, crear, comunicar, y calcular mediante el uso de materiales escritos e impresos relacionados con distintos contextos" (p. 16). Según lo planteado, el proceso de alfabetizar implica un aprendizaje continuo en el que se obtienen herramientas que capacitan a los participantes para desarrollarse como actores de la sociedad en la que se encuentran al tiempo que crecen como personas en sus ámbitos profesionales y personales. Dentro de esta misma línea de pensamiento se encuentra la concepción que Ortiz (2007) enuncia sobre alfabetización, al describirla como:

Un proceso educativo que crea un ambiente propicio para que los estudiantes desarrollen sus competencias comunicativas escritas, asumiendo la lectura y la escritura como una oportunidad constante de aprendizaje y crecimiento que les permite descubrir el pensamiento de otros, entender la divergencia, ampliar, confrontar y comunicar su visión del mundo y de la vida. (p. 16)

Crystal (2011), a su vez, define el término alfabetización como la capacidad de comprender y manejar distintos dominios culturales y tecnológicos. El autor se refiere en esta conceptualización al hecho de que cada día el término abarca más y más habilidades, por ejemplo, el uso eficiente de los computadores, teléfonos inteligentes, conocimiento básico de más de un idioma, así como de terminología y modismos globales. Dentro de la misma línea, Richards y Schmidt (2013) relacionan las prácticas y el comportamiento alfabetizado con la socialización y el desenvolvimiento de los individuos en la sociedad por medio del uso de la escritura. Desde esta perspectiva una persona alfabetizada sería aquella que es capaz de comprender, producir y básicamente interactuar con todos los elementos y en todas las situaciones dentro de la sociedad a la que pertenece. No se trata, entonces, de saber leer y escribir, o de expresar y comprender mensajes; se trata de ser capaz de interpretar y mane- jar la información que produce la comunidad global, en otras palabras, ser estratégicamente competente en todos los contextos actuales de comunicación.

No es imprudente, entonces, decir que es preciso mudar el enfoque de la alfabetización recorriendo el camino del racionalismo crítico y el desaprendizaje para incluir en él las destrezas requeridas en la producción y procesamiento de conocimiento hoy en día.

\section{De la reflexión crítica al desaprendizaje}

Ya se ha planteado que a diferencia del lenguaje oral, la escritura requiere de un proceso de instrucción formal, y que las convenciones ortográficas, sintácticas y pragmáticas rigen el discurso escrito según cada cultura y deben ser puestas en práctica para que la producción sea aceptada. Este proceso de instrucción abarca tanto la lectura como la escritura y se va adaptando, o debería adaptarse, a los cambios culturales y tecnológicos de cada comunidad. Ya lo afirmaban Cassany y Morales (2008) cuando explicaban que a pesar de que puedan existir destrezas cognitivas generales en el aprendizaje de la lengua escrita no se pueden dejar de lado el impacto de las formas y costumbres propias de cada comunidad de escritores.

Esto implica que si bien hace veinte años los niños tenían sus primeros contactos con la lengua escrita a leer en las cartillas y los tradicionales libros de silabeo, mientras que la escritura estaba ligada a la caligrafía y la estética, hoy día no es de extrañar que los primeros eventos de lectura y escritura se hagan en una computadora o tabletas presionando teclas y leyendo pantallas, interpretando imágenes y haciendo formas libres y dibujos con los dedos, en lugar de hacerlo con un libro y un lápiz, aunque este hecho no necesariamente ha afectado la visión de proceso y producto de escritura dentro de las aulas de clase.

Cabe entonces preguntarse, ¿Qué ha cambiado en la visión de la enseñanza de la lengua 
escrita? ¿Se ha modificado la concepción de alfabetización que caracteriza las prácticas sociales de los docentes? ¿Ha evolucionado la forma de enseñar y aprender a escribir, o simplemente se incluyen los computadores como un accesorio y no como un verdadero instrumento de comunicación?

Sin duda es en el aprendizaje de la escritura donde se puede percibir en mayor grado el cambio impulsado por la tecnología, si bien hay principios funcionales y ortográficos que se mantienen, el proceso de escritura tendría que ser objeto de un fenómeno de desaprendizaje, esto entendiendo primeramente, que aprender es la "construcción y reconstrucción continuas de la realidad" (Guidano citado en Andrade, 2005 , p. 2), y por consiguiente para desaprender debe transitarse por una ruta similar pero desmontando los conceptos, teorías, convenciones y estructuras que culturalmente se consideraban como dogmas absolutos para adaptarlos a las nuevas necesidades y los nuevos procederes de los individuos. En este sentido, Andrade (2005) continúa afirmando que desaprender es reconocer que los seres humanos son movimiento $y$ de este modo deben deshacerse y rehacerse según los cambios que provengan de cuestionar el entorno.

Ya Popper (2008) hablaba de la importancia de cuestionar la realidad en base a las dinámicas propias de la sociedad así como de los datos recolectados empíricamente partiendo de una contrastación deductiva, en lo que pasó a denominarse como racionalismo crítico. Es este cuestionamiento de los conceptos existentes lo que obliga a re-evaluar las convenciones históricas sobre el aprendizaje de la lengua escrita, es decir, es el mismo dinamismo, las necesidades y exigencias de los nuevos tiempos lo que lleva a generar cambios en los presupuestos referentes a las prácticas sociales de alfabetización de las escuelas. Si bien los niños siguen percibiendo la necesidad de aprender a leer y escribir en base a la utilidad que tenga para ellos esta forma de comunicación, los aprendices de hoy día no perciben la escritura ni hacen uso de ella de la misma manera que hace diez años, por lo que se podría asumir que el método de enseñanza de hace una década llegaría a ser ineficiente e insuficiente para la generación actual.

Es importante acotar el hecho de que los textos y los actos que se derivan de ellos son el reflejo del pensamiento del individuo. Se piensa y se altera la forma de pensar en la medida en que cambia el vocabulario, por lo que la forma en la que las nuevas nociones virtuales intervienen en la manera en que se expresan las personas tiene peso en el comportamiento; la palabra altera la conciencia y por tanto los procesos de aprendizaje, tal y como lo establece Andrade (2004). Si en un plano ontológico; siguiendo las afirmaciones de Govea (2016), el estudio de las prácticas sociales, los roles desempeñados por los humanos y la complejidad de pensamientos en sus mentes nos llevaría a reforzar la idea anterior de cómo los cambios en las formas de expresión han tenido influencia en las actuaciones e incluso las interacciones del seres humanos, mucho más se refuerza la necesidad de iniciar una deconstrucción crítica de las prácticas sociales de alfabetización en el país.

Se busca, entonces, partir de una revisión de las teorías relativas a la alfabetización con el objeto de establecer un sistema teórico con el cual se contrastarán los datos que se recolecten de la realidad en estudios posteriores. Esto con el fin de hacer una comparación para de este modo determinar las equivalencias, compatibilidades o incompatibilidades que en última instancia llevarán a enunciar una concepción de alfabetización que esté más acorde con los actuales contextos comunicacionales.

Lo que se busca no es más que someter las actuales concepciones sobre la alfabetización a un proceso de falsación, pues tomando las palabras de Echeverría (1989) este proceso es en efecto lo que genera cambios y avances dentro de las comunidades de conocimiento. Siguiendo con los planteamientos del autor, se tiene que en el sistema actual de educación en Venezuela se 
trabaja con unas nociones y unas metodologías de alfabetización que han sido heredadas a través de los años y provenientes de realidades que no necesariamente son compatibles con la actual situación del país, pero a partir de un cuestionamiento consciente y una deconstrucción crítica de las prácticas sociales relacionadas con la lengua escrita se pueden activar modificaciones que lleven a una verdadera apropiación del carácter cognitivo y comunicativo de la escritura.

En este orden de ideas, son las premisas del racionalismo crítico las que se pretenden seguir para plantear esta revisión teórica de acuerdo con los planteamientos de Popper. Este autor concebía el racionalismo crítico como una especie de motor que impulsa a desechar la inmutabilidad del conocimiento científico. En efecto, si se toma evidencia empírica proveniente de, por ejemplo, las aulas de bachillerato, se podría encontrar innumerables fallas de los estudiantes a nivel de la lecto-escritura en lo referente a las micro y macro estructuras de los textos así como la comprensión crítica de los mismos. Más aun, se encontraría que la mayoría de los estudiantes no sienten inclinación alguna por el lenguaje escrito más allá del contexto escolar o de lo que pueda llegar a serle útil dentro del espacio de las redes sociales (Pineda, Lugo, \& Arévalo, 2016). Solo esta evidencia, sería suficiente para proponer que el actual concepto de alfabetización está dando pie a prácticas erróneas por lo que no es precipitado afirmar que está obsoleto.

Sin duda, los supuestos enunciados por Popper (2008) no están alejados de las máximas relacionadas con el desaprendizaje. El desaprendizaje se define como el acto de recorrer el camino marcado por supuestos conceptuales, hasta los momentos inamovibles, para reconstruir tales supuestos según las nuevas necesidades y realidades. Andrade (2005), indica que "el conocimiento es consciencia de la heterogeneidad biológica, psíquica, sensorial, social y espiritual, del sujeto cognoscente" (p. 4). En un plano gnoseológico se afirmaría que el conocimiento es producto del hombre y su interacción con el mundo, por consiguiente, es él mismo el responsable y artífice de las transformaciones cognitivas que puedan o no darse de la aceptación o rechazo de los parámetros establecidos en su campo de acción.

Evidentemente, el conocimiento producto del lenguaje y configurado a través de las palabras solo puede ser transformado o reaprendido por medio del renombramiento de los conceptos. La misma Andrade (2005) establece que "el sujeto es capaz de desprenderse de todas sus palabras y reempalabrarse" (p. 8) cuando pasa por el proceso de desechar lo que Cegarra y Rodrigo (2004) llaman conocimiento obsoleto o engañoso.

Se trata, entonces, no solo de recuperar saberes ya enunciados por otros sino de ir construyendo nuevos saberes y nuevas formas de pensar por medio del cuestionamiento crítico e intencional de lo que ya existe. Piaget (citado en Quiñónez, \& Vélez, 2004) denominaba a esto como "desequilibrio", en el que de manera casi instintiva el individuo formaba una serie de resistencias dirigidas a proteger sus sistemas de creencias. Sin embargo, este mismo conflicto cognitivo, impulsado por inconformidades o vacíos en el saber es lo que lleva a la construcción de nuevas representaciones sociales y a la transformación de realidades, en otras palabras lleva a la falsación.

\section{Metodología y resultados}

Este trabajo se origina a partir de una revisión documental sistemática de las fuentes de información oficial disponibles en Venezuela en relación a las prácticas de alfabetización dentro y fuera del contexto escolar. No está de más destacar que la investigación documental parte de la recolección, organización, análisis e interpretación de información proveniente de fuentes bibliográficas sean estas físicas o electrónicas (Colls, 1994). En este sentido se procedió a delimitar el tema, acopiar la información, organizar la data y elaborar un esquema conceptual para 
finalmente completar el análisis de lo encontrado, tal y como lo recomienda Morales (2003).

En este orden de ideas, habiendo delimitado el tema como el concepto de alfabetización en dentro del Estado venezolano se comenzó con una revisión en los portales web institucionales y las publicaciones oficiales, como el documento de la Misión Robinson (2005), para pasar seguidamente a una contrastación de la información obtenida de esta fuente con lo manejado en los documentos oficiales obtenidos en la Zona Educativa del estado Falcón, entre ellos principalmente el Currículo del Subsistema de Educación Primaria del año 2007, las Orientaciones Pedagógicas del Ministerio del Poder Popular para la Educación (MPPE) 20172018 y la Memoria y cuenta del Ministerio del Poder Popular para la Educación (2013).

A partir de esta recolección, y habiendo elaborado el esquema conceptual de la data, se derivó un análisis en el que se pudo evidenciar varios aspectos. En primer lugar estuvo claro que no existe en las fuentes de información oficiales un concepto actual y unificado sobre la alfabetización fuera de la descripción metodológica de la Campaña Bolivariana de Alfabetización puesta en práctica a partir del año 1999 la cual estaba dirigida solo a la población adulta y su basamento principal partía de las directrices concebidas por el método cubano "Yo sí puedo".

Cabe acotar con respecto a este punto en particular que, fuera de las cifras contradictorias publicadas por la UNESCO y por el Gobierno venezolano, según las cuales en 2015 la UNESCO afirmaba que el índice de alfabetismo en Venezuela es $95.4 \%$ mientras que la escaza información oficial disponible predica que la tasa de analfabetismo en Venezuela es 0; no existe información reciente y concreta con respecto a este programa, ni archivo del personal docente a cargo de la misma con el fin de hacer seguimiento.

En segundo lugar, otro aspecto a destacar es que la carencia de un concepto oficial de alfabetización emanado del MPPE deriva por consi- guiente en que tampoco exista un método adaptado a las necesidades específicas de la población venezolana por lo que queda a cargo de cada docente en particular la decisión del método más apropiado a aplicar, lo que puede traer inconsistencias en el desarrollo de la lectura y escritura entre la población estudiantil, incluso dentro de una misma institución (Pineda et al., 2016).

Así mismo, fue evidente en esta revisión que las últimas reformas curriculares ocurridas en Venezuela estuvieron más orientadas a reformar la estructura general de las asignaturas cursadas en la educación primaria y secundaria, la configuración de la comunidad educativa y las temáticas a abordar más no el método de enseñanza a ningún nivel (MPPE, 2017).

Finalmente a partir de esta revisión se pudo elaborar una ruta histórica de los programas de alfabetización en Venezuela lo que sirvió para ilustrar más detalladamente la evolución del concepto desde el punto de vista de las políticas del Estado venezolano.

\section{Un recorrido histórico de la alfabetización en Venezuela}

Los programas orientados a enseñar la lengua escrita tanto a adultos como a niños en entornos escolares y extra-escolares que ha implementado en gobierno venezolano han pasado por una serie importante de transformaciones en cuanto a concepto y metodología desde el siglo pasado. De hecho, en Venezuela la enseñanza de la lectura y la escritura ha sido atendida en programas destinados a adolescentes y adultos fuera del ambiente escolar desde hace por lo menos 58 años. Una exploración de esta naturaleza acerca de estos programas extra-escolares puede dar una idea del concepto de alfabetización que se tiene dentro del Estado venezolano y como ha ido cambiando para adaptarse a los devenires políticos y a las directrices del Estado.

Por ejemplo, en 1958 luego de la caída de la dictadura de Marcos Pérez Jiménez, se crea el programa Abajo Cadenas, el cual se definió 
como un programa para la "redención cultural de la población analfabeta" (p. 7) y contaba con dos objetivos, uno cultural: "La elevación de las masas laborales para incorporarlas de inmediato al desarrollo cultural, técnico y económico que exigen las circunstancias en que vive la nación" (p. 9) y otro político:

Desarrollar un sano nacionalismo en la defensa común de nuestros intereses y valoración de lo nuestro; es decir, un nacionalismo constructivo que acondicione en la conciencia del venezolano de hoy el sentimiento de superar nuestra deprimente condición de pueblo; procuraremos crear una conciencia eminentemente nacional en los venezolanos, arraigando en su sentir y en su pensar ideales venezolanistas que lo conduzcan a exaltar nuestros valores históricos, a defender nuestro patriotismo económico y a promover un movimiento conjunto de todos los sectores nacionales en el sentido de erradicar la miseria y la ignorancia. La Campaña buscará sentar las bases de una nación verdaderamente independiente en el futuro. (Misión Robinson, 2005, p. 10)

Aquí ya se describía el analfabetismo como fuente de miseria e ignorancia, así como de desarraigo cultural, en este programa el analfabetismo priva al ciudadano de la ejecución de sus derechos y deberes con la nación, en actividades productivas y políticas. Para abordar esto se usaba el método de instrucción de la palabras normales, el cual no es más que la asociación de una palabra básica o generadora con una imagen y seguidamente descompuesta en sílabas y letras. Esta palabra debe ser de uso frecuente para los aprendices y estar directamente ligada a su contexto. La alfabetización en este programa se percibe como un proceso mecánico que no estimulaba la creación independiente ni la comprensión del texto como instrumento de expresión y crecimiento personal; para el programa Abajo Cadenas, el manejo de la lengua escrita es un medio para insertarse en el desarrollo económico del país.
Con este paso inicial los programas de alfabetización se fueron transformando, y así en 1963 se crea el Consejo Nacional de Alfabetización cuyo objetivo era "integrar los programas de alfabetización y de extensión cultural, con los de extensión agrícola o capacitación laboral, promoción social y saneamiento ambiental" (Misión Robinson, 2005, p. 27). Nuevamente la práctica de la lecto-escritura es vista y manejada desde las actividades económicas y no desde la interacción social y la expresión cultural. Se pretende en este programa enseñar a leer y escribir a futuros trabajadores y no a individuos en busca de medios de expresión e interacción. Ya para 1979 se crea la Asociación Cultural para el Desarrollo o ACUDE que fue una asociación sin fines de lucro ideada por el sector privado para la que la educación es "un proceso de toda la vida que trasciende la sola experiencia escolar y puede ser desarrollada por individuos o grupos familiares" (Misión Robinson, 2005, p. 30).

El programa ACUDE cambia la concepción de lenguaje escrito como algo que se aprende para poder trabajar a una actividad que está ligada a todos los contextos del día a día, en la vida ciudadana y familiar, aunque no deja de estar relacionada con las actividades laborales y económicas. Así pues, es durante la aplicación de ACUDE que se introduce el uso de medios audiovisuales (grabaciones y textos impresos) para la enseñanza del lenguaje escrito enfocando sus mensajes en aspectos de convivencia, salud, higiene y desempeño laboral.

Posterior a esto, el INCE (Instituto Nacional de Capacitación y Educación) en 1985 comienza a implementar el Programa de Alfabetización Nacional, primero con la Labor Alfabetizadora del INCE y seguidamente en 1989 con el Curso de Alfabetización básica que contemplaba como objetivos:

Alfabetizar a nivel básico a trabajadores actuales y potenciales. Desarrollar una clara comprensión de los valores nacionales y de las exigencias de la vida moderna acordes con el proceso de desarrollo del país. Contribuir al 
ajuste y desarrollo de la personalidad de los participantes como miembros de una comunidad urbana en una democracia. Desarrollar el pensamiento reflexivo, especialmente en lo concerniente al enfoque de relaciones humanas, problemas sociales y problemas del trabajo. (Misión Robinson, 2005, p. 57).

Se continúa con la visión que ya se traía, de la alfabetización como instrumento de inserción al campo laboral y se seguirá con esta hasta la implementación del Plan nacional de atención a la población adulta con necesidades de aprendizaje básico - Comisión nacional de alfabetización de 1994, que en este caso fue manejado por el Ministerio de Educación con el cual se da una visión más humanista y de construcción del conocimiento.

En 1999 se comienza con la Campaña Bolivariana de Alfabetización que en 2003 daría origen a la Misión Robinson, un programa de tres etapas: Alfabetización, Batalla por el Sexto Grado, y Círculos de lectura, que estuvo destinado a:

Erradicar el analfabetismo y alcanzar la prosecución al sexto grado de educación primaria en jóvenes y adultos a nivel nacional e internacional garantizando su inclusión política, social y productiva con el propósito de conseguir mayores niveles de desarrollo y bienestar en los grupos sociales más necesitados del país. (Memoria y cuenta del Ministerio del Poder Popular para la Educación, 2013, p. 45)

En este programa el analfabetismo es fuente de exclusión y privativo del desarrollo y bienestar de grupos sociales, por lo que es política del Estado atacarlo.

Con esto en mente, se utiliza el programa "Yo sí puedo" ideado por el Gobierno de Cuba en el cual se implementan medios audiovisuales (Videos, grabaciones, cartillas) para adiestrar en un número específico de clases, divididas en tres etapas. Así, se tienen diez clases en la primera etapa y 42 en la segunda, al final de las cuales los participantes deben ser capaces de escribir su nombre, reconocer y escribir los números de
1-30, y construir oraciones simples. Al culminar la tercera etapa de la Misión Robinson, los aprendices deben ser capaces de comprender lecturas y redactar textos simples. A estas alturas las clases (11-12) son dirigidas a monitorear problemas con la grafía y la comprensión de la lectura a nivel literal.

Se puede observar de este modo cómo la noción de analfabetismo, fuera del contexto de la educación formal, ha ido cambiando a través de los años según los programas que ha implementado el Estado para atender este fenómeno, aunque siempre resalte dentro de su concepción el carácter excluyente y económicamente improductivo de la condición de analfabeta.

Es pertinente afirmar que estas premisas son igualmente aplicables a las prácticas de alfabetización dentro de los entornos de educación primaria, como ya se puntualizó, no existe en Venezuela un concepto ni una práctica unificada de alfabetización que emane del Ministerio del Poder Popular para la Educación, quedando bajo la responsabilidad y preparación profesional de los maestros las estrategias para la enseñanza de la lectura y escritura dentro del aula.

Así pues, al no existir un consenso en torno a lo que deben ser las prácticas de alfabetización dentro de la escuela, tampoco hay una ruta uniforme adaptada a la realidad venezolana que brinde a los niños las herramientas para apropiarse de la lengua escrita y usarla como vehículo para su desarrollo personal y, por ende, para contribuir al progreso nacional. Por el contrario, se evidencia que los egresados de educación primaria y secundaria van arrastrando problemas de comprensión y producción escrita que, en la mayoría de los casos, les impide participar e interactuar eficientemente dentro de su comunidad a través de la escritura.

En este orden de ideas, podría considerarse como un acuerdo actual que si un individuo alfabetizado es aquel que es capaz de socializar información producida por la sociedad en diferentes medios, entonces un analfabeta sería quien por sus capacidades o por falta de ellas ve limitado su 
campo de acción y su interacción con la información escrita en su entorno. En esta línea los conceptos y prácticas de alfabetización deben estar orientadas a proveer a las personas de estas herramientas para insertase activamente en la sociedad, por lo que trasciende del aprendizaje de la lectura y la escritura para posicionarse en la de aprendizaje de habilidades de dominio e interacción con el mundo a través de la lectura y la escritura (Leal, 2016).

\section{Consideraciones finales}

Si la alfabetización implica un proceso de crecimiento personal, el desaprendizaje de este concepto a través de la contrastación deductiva, implica hacerse cargo de que ese crecimiento esté adaptado a los tiempos que vive cada individuo y al grupo social del que forma parte, lo cual es un proceso que nunca debe detenerse tal como las dinámicas sociales tampoco se detienen.

A través de los años el concepto de alfabetización en Venezuela, ha estado alejado del verdadero carácter comunicativo de la práctica de la lengua escrita, orientándose más hacia el aprendizaje de capacidades para la conducción de actividades económicas. Esto ha traído como consecuencia que los individuos perciban la escritura o la lectura como una obligación laboral o educativa, como algo que se lleva a cabo para cumplir con algún requisito o asignación y no como un medio para expresarse y conectarse con su entorno.

Esta exploración inicial de los principales aspectos teóricos y epistemológicos relacionados con la alfabetización resaltan la necesidad de continuar en el estudio de esta práctica desde el punto de vista del desaprendizaje y la contrastación crítica de conceptos, con el fin de transitar el camino que llevará a la elaboración de una nueva concepción que propicie prácticas alfabetizadoras a nivel escolar y extra-escolar que cultive escritores y lectores, y no simplemente individuos que codifiquen y decodifiquen su lengua.

El fin último es usar las nociones aquí planteadas como hoja de ruta inicial para una futura indagación más profunda en las prácticas alfabetizadoras en Venezuela, indagación que pueda iniciar una transformación de las conductas actuales hacia otras en las que se aprecie en su totalidad todo lo que los seres humanos pueden lograr a través de la lengua escrita.

\section{Referencias bibliográficas}

Andrade, R. (2004). Hacia una gnoseología del desaprendizaje consciente. Los tatuajes de la palabra y la consciencia de los tatuajes. Fermentum, 14(39), 28-52.

Andrade, R. (2005). Hacia una gnoseología del desaprendizaje consciente: Principios para desaprender en el contexto de la complejidad. Revista Electrónica de Investigación Educativa, 7(2).

Brown, D. (2007). Principles of language learning and teaching. Inglaterra: Longman.

Cassany, D. (1999). Construir la escritura. Barcelona: Paidós Iberica.

Cassany, D. (2003). Escritura electrónica. C \& E, 15(3), 239-251.

Cassany, D., \& Morales, O. (2008). Leer y escribir en la universidad: Hacia la lectura y la escritura crítica de géneros científicos. Barcelona: Universitat Pompeu-Fabra.

Cegarra, J., \& Rodrigo, B. (2004). Desaprendizaje individual: Un paso previo a la creación del capital relacional. Cuad. Adm., 17(27), 11-32.

Colls, M. (1994). Introducción a la investigación documental. Mérida: Consejo de publicaciones de la ULA.

Crystal, D. (2011). A dictionary of language. Chicago: University of Chicago Press.

Echeverría, J. (1989). Introducción a la metodología de la ciencia. Barcelona: Barcanova.

Ferreiro, E., \& Gómez, M. (2002). Nuevas perspectivas sobre el proceso de lectura y escritura. México: Siglo XXI editores.

Ferreiro, E., \& Teberosky, A. (1991). Los sistemas de escritura en el desarrollo del niño. México: Siglo XXI editores.

Franco, A. (2000). Pragmática y gramática comunicativa. Utopía y praxis latinoamericana, 5(9), 81-93. 
Govea, L. (2016). Significaciones y prácticas de literacidad de los docentes en el ámbito universitario como base para la creación de una metodología de lectura en su contexto. Tesis para optar al grado de Doctor en Educación en la Universidad Pedagógica Experimental Libertador Instituto Pedagógico Rural "Gervasio Rubio".

Harmer, J. (2000). Teaching English lenguaje [Enseñando el idioma inglés]. México: McGraw-Hill.

Ministerio para el poder popular para la educación (2013). Memoria y cuenta. Caracas.

Ministerio para el poder popular para la educación (2007). Currículo del Subsistema de Educación Primaria. Caracas.

Ministerio para el poder popular para la educación (2017). Orientaciones Pedagógicas Año Escolar 2017-2018. Caracas.

Morales, O. (2003). Fundamentos de la investigación documental y la monografía. Manual para la elaboración y la presentación de la Monografía. Mérida: Consejo de Publicaciones de la ULA. Misión Robinson (2005). Yo sí puedo. Educere, 9(28), 9-18. (https://bit.ly/2LOJ80p) (2016/10/08).

Ortiz, M. (2007). La alfabetización inicial en la escuela. Caracas: IESALC-UNESCO.

Pineda, C., Lugo, M., \& Arévalo, J. (2016). Diagnóstico para el proyecto "La escuela y la lengua escrita”. Proyecto de Investigación avalado por el Decanato de Investigación UNEFM. Coro, Venezuela: Universidad Nacional Experimental Francisco de Miranda.

Popper, K. (2008). La lógica de la investigación científica. Madrid: Tecnos.

Quiñónez, J., \& Vélez, C. (2004). Algunas condiciones pedagógicas para la formación de la investigación en la universidad. Actualidades educativas en investigación, 4(1), 2-27.

Richards, J., \& Schmidt, R. (2013). A dictionary of language teaching and applied linguistics. Nueva York: Routledge. 